\title{
Разложение этопозида в водно-органических растворах и разделение продуктов его деструкции методом ВЭЖХ
}

\author{
Голубицкий Г.Б., Островский К.П., Гельперина С.Э. \\ ООО «Технология лекарств», Химки
}

Поступила в редакцию 14.03.2018 г.

DOI: https://doi.org/10.17308/sorpchrom.2018.18/562

Впервые изучено влияние ацетонитрила и диметилсульфоксида на скорость разложения этопозида в щелочном водно-органическом растворе. Органические растворители замедляют деструкцию, что связано с изменением диэлектрической проницаемости растворов. Влияние диметилсульфоксида на скорость реакции и форму пиков этопозида значительно слабее в связи с его более высокой диэлектрической проницаемостью и полярностью по сравнению с ацетонитрилом. Подобраны условия разделения этопозида и продуктов его разложения при помощи обращенно-фазной ВЭЖХ. Сделано предположение о химической природе продуктов разложения этопозида в щелочной среде. Показаны возможности использования подвижной фазы, содержащей перхлорат аммония, для повышения чувствительности анализа.

Ключевые слова: этопозид, щелочное разложение, влияние органического растворителя на скорость разложения, разделение методом обращенно-фазной ВЭЖХ, летучие компоненты подвижной фазы, пропускание в коротковолновой области УФ-спектра.

\section{Decomposition of etoposide in aqueous-organic solutions and separation of its degradation products by HPLC}

\author{
Golubitsky G.B., Ostrovskiy K.P., Gelperina S.E. \\ LTD «Drugs Technology», Khimki
}

The purpose of this study is optimization of HPLC analysis conditions for etoposide and its decomposition products and application of the mobile phase based on volatile, transparent in the short wavelength ultraviolet region of the spectrum components to approach the way to unify the chromatography analysis.

We determined kinetic parameters for etoposide decomposition in the alkaline medium. We have studied for the first time the effect of acetonitrile and dimethyl sulfoxide on the rate of etoposide decomposition in the alkaline aqueous-organic solution. We showed that organic solvents inhibit the degradation due to the changed dielectric constant of the medium. The effect of dimethyl sulfoxide on degradation and peak shape is much weaker, because of its higher dielectric constant and polarity compared to acetonitrile.

The separation conditions of etoposide and its decomposition products by means of reverse-phase HPLC are selected. We compared the retention time received for degradation products at different $\mathrm{pH}$ of the mobile phase with the data from literature, and the assumption is made about the chemical nature of the decomposition products of etoposide in the alkaline medium. We showed the possibilities of use of mobile phase containing water, acetonitrile, perchloric acid and ammonia to increase sensitivity of detection. This approach is perspective for other drugs of different chemical nature. 
Keywords: etoposide, alkaline decomposition, effect of organic solvent on the rate of decomposition, the separation by reversed-phase HPLC, volatile components of the mobile phase, transmission in the short-wave region of the UV spectrum.

\section{Введение}

Этопозид (ЭТ)- высокоэффективное лекарственное средство, обладающее противоопухолевой активностью [1]. При превышении установленных доз возможно проявление токсических свойств ЭТ, поэтому разработка точных и чувствительных методик определения этого вещества в объектах биологической природы и в лекарственных препаратах чрезвычайно актуальна. Для количественного определения ЭТ и продуктов его разложения наиболее перспективны хроматографические методы.

Согласно литературным данным, подходы к анализу объектов, содержащих ЭТ, разнообразны. В большинстве случаев анализ ведут обращенно-фазной ВЭЖХ; публикации об использовании для анализа ЭТ нормально-фазной ВЭЖХ нами не найдены. В ряде случаев для элюирования применяли подвижные фазы (ПФ) на основе смесей ацетонитрила с водой [2], либо метанола с водой [3], не содержащие солей. Такие условия не являются оптимальными, что подтверждается приведенными результатами. Так, при времени удерживания ЭТ около шести минут на колонке длиной 150 мм с размером зерен сорбента 5 мкм ширина пика у основания составляет около двух минут, что свидетельствует о низкой эффективности разделения [3]. В качестве буферной составляющей ПФ чаще всего используют растворы натрия ацетата [4,5], натрия цитрата [6], уксусной [7-9] или муравьиной [10-13] кислот. Фосфатные буферные растворы добавляли в ПФ в работах [14-19]. Величина рН буферных составляющих ПФ в цитируемых работах составляла 2.0-7.0, но значение данного параметра в большинстве случаев не обосновано. Так, отмечается, что некоторые продукты разложения ЭТ обладают кислотными свойствами, при выбранном рН ПФ $(6,5)$ диссоциированы и слабо удерживаются. Для повышения их удерживания в ПФ вводят тетрабутиламмония бромид [19]. Не ясно, почему не использован более простой путь решения данной проблемы, обеспечивающий больший срок службы сорбента - снижение $\mathrm{pH}$ ПФ. В другой работе в ПФ добавляли анионный ион-парный реагент - 1-гептансульфоновую кислоту, обычно используемую для повышения удерживания веществ основной природы. Обоснования необходимости данного компонента в публикации нет [16]. Некоторые исследователи полагают, что при использовании ПФ с рН, близкими к нейтральным, или слабощелочным, может происходить эпимеризация - переход ЭТ (А) в фармакологически неактивную цис-форму (B) $[8]$.
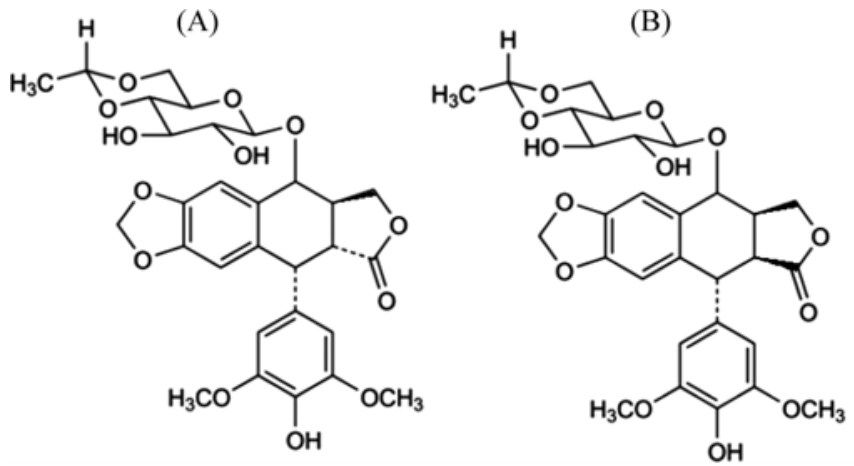

Однако другие работы эту информацию не подтверждают - эпимеризацию ЭТ в ПФ с рН 6,0 $\div$ 7,0 не наблюдали [19]. 
Для детектирования ЭТ используются УФ-[2-5,7,8,10,14,18,19], массспектрометрические [11-13], электрохимические $[6,15,17]$, флуориметрические $[9,16]$ детекторы. Максимальную универсальность и чувствительность обеспечивает тандемное масс-спектрометрическое детектирование, необходимое для идентификации продуктов разложения при исследовании метаболизма, стабильности лекарственных препаратов, при анализе биологических и природных объектов, когда уровень содержания ЭТ в пробах низок. Предел определения ЭТ данным методом, составляет $7.2 \cdot 10^{-6}$ мкг $/ \mathrm{cm}^{3}$ [13]. Высокую чувствительность обеспечивает флуориметрическое и электрохимическое детектирование, хотя эти способы значительно уступают по данному показателю масс-спектрометрии (пределы определения ЭТ от 0.03 [9] мкг/ $\mathrm{cm}^{3}$ и от 0.0075 [6] мкг $/ \mathrm{cm}^{3}$ соответственно). Менее чувствительны методики, использующие УФ-детектирование: предел определения от 0,04 мкг/мл достигнут благодаря большому объему дозируемой пробы (100 мкл) [18]. Анализ литературных данных позволяет сделать вывод, что возможности повышения чувствительности определения ЭТ с наиболее распространенным в фармацевтическом анализе УФдетектированием исчерпаны не полностью.

Стабильность ЭТ в водных растворах $[8,19]$, в твердом состоянии [20] и в составе лекарственных препаратов [5,7] изучена подробно. Исследовано влияние температуры $[7,19,20], \mathrm{pH}[8,19]$, природы буфера и ионной силы растворов [19], материала упаковки или присутствия других цитостатиков [7]. Публикаций о влиянии органических растворителей на стабильность ЭТ в растворе нами не найдено. Однако этот фактор является чрезвычайно важным для оптимизации условий пробоподготовки при анализе лекарственных препаратов.

Цели настоящего исследования - обоснование состава растворителя, используемого для анализа препаратов, содержащих ЭТ, а также подтверждение преимуществ использования ПФ ацетонитрил - вода - перхлорат аммония для хроматографического разделения ЭТ и продуктов его разложения с УФ-детектированием.

\section{Эксперимент}

Использовали жидкостный хроматограф Prominence LC-20 с автоматическим дозатором SIL-20AC и диодно-матричным детектором SPD-M20A (Shimadzu), а также pH-метр Seven Compact S20 с комбинированным стеклянным электродом (Mettler Toledo).

ЭТ и продукты его разложения разделяли на колонке размером 4.6×100 мм с защитной предколонкой 20×4.6 мм, заполненных сорбентом XBridge Shield RP18 c размером частиц 3.5 мкм (Waters),

Для приготовления ПФ использовали смеси ацетонитрила с водными растворами, содержащими 0.5 об.\% хлорной кислоты, $\mathrm{pH}$ которых доводили до необходимого значения водным раствором аммиака с потенциометрическим контролем. Расход ПФ составил $1.0 \mathrm{~cm}^{3} /$ мин, объем проб 20 мкл, температура колонок $40^{\circ} \mathrm{C}$, температура образцов $5^{\circ} \mathrm{C}$, длина волны детектирования 205 нм, соответствующая коротковолновому максимуму поглощения ЭТ.

Использовали сверхчистую воду из установки Advantage A10 (Millipore), peактивы фирмы PanReac - ацетонитрил UHPLC Supergradient, гидроксид натрия и фирмы Sigma Aldrich - водный раствор аммиака (25 масс.\%), хлорную кислоту (70 масс.\%). Все использованные реактивы, кроме ацетонитрила - reagent grade), ЭТ фармакопейного качества (содержание основного вещества 99.6 \% согласно паспорту лекарственного средства, производство - Китай). 
Для приготовления растворов ЭТ при изучении их стабильности и (или) разделения компонентов в мерную колбу вместимостью 100 мл помещали аликвоту исходного раствора ЭТ в ацетонитриле или ДМСО, добавляли недостающее количество соответствующего растворителя и водного раствора реагента, влияние которого на стабильность исследовали. Полученный раствор перемешивали, доводили его объем до метки водой и перемешивали. Сразу после приготовления анализировали полученный раствор методом ВЭЖХ при условиях, указанных в подписях к рисунку 2. При исследовании кинетики разложения ЭТ продолжительность одной инжекции определяла интервал между точками на оси времени.

\section{Обсуждение результатов}

ПФ состава ацетонитрил - вода - перхлорат аммония обеспечивает длительную бесперебойную работу хроматографа и колонок. Это обусловлено высокой растворимостью солей $\mathrm{HClO}_{4}$ в водных и водно-ацетонитрильных растворах, что допускает немедленный переход от подвижной фазы к 100\% ацетонитрила без образования осадка в трубках и колонках. Ранее была показана перспективность использования данного состава ПФ для ВЭЖХ-анализа рисперидона и его смесей с продуктами разложения [21].

Выбор оптимальных условий детектирования. При анализе ЭТ и его препаратов данный состав ПФ имеет и другое преимущество, связанное с его прозрачностью в коротковолновой УФ-области спектра. ЭТ имеет в УФ-области два основных максимума поглощения - при 205 и 284 нм. Прозрачный в дальнем ультрафиолете растворитель позволяет измерять оптическую плотность при 205 нм (рис. 1).

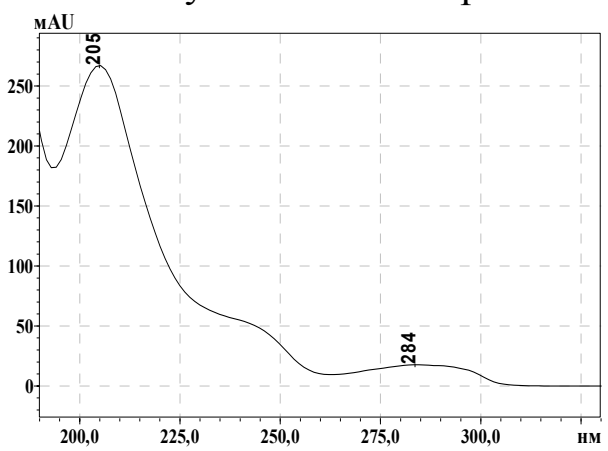

Рис. 1. УФ-спектр поглощения этопозида в ПФ, полученный при помощи диодно-матричного детектора в режиме on-line.

По спектру ЭТ также видно, что величина УФ-поглощения ЭТ при 205 нм примерно в 10 раз выше, чем при 285 нм и, как следствие, использование коротковолнового максимума поглощения на порядок повышает чувствительность детектирования. При количественном определении продуктов разложения ЭТ выигрыш в чувствительности зависит от спектральных и хроматографических характеристик этих продуктов. Конкретные значения могут быть определены по хроматограммам раствора, содержащего ЭТ и продукты его разложения с детектированием при 205 и 284 нм (рис. 2).

Результаты расчета величин шума базовой линии и соотношений сигнал/шум для пиков ЭТ и некоторых продуктов его разложения представлены в табл. 1. Согласно этим значениям, чувствительность детектирования ЭТ и продуктов его разложения при 205 нм до 20 раз выше, чем при 284 нм. Учитывая, что концентрация

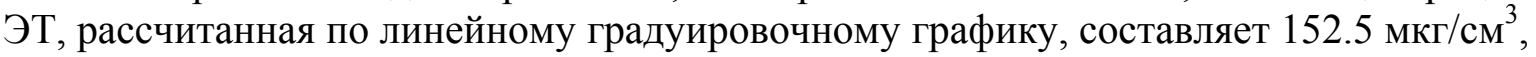
а величина S/n при этом равна 26600, концентрация данного вещества, соответст- 
вующая значению $\mathrm{S} / \mathrm{n}=10$, равна 0.057 мкг $/ \mathrm{cm}^{3}$. Это значение соответствует пределу количественного определения ЭТ при используемых условиях анализа и не уступает более чувствительным методам - электрохимическому [6] и флуориметрическому [9].
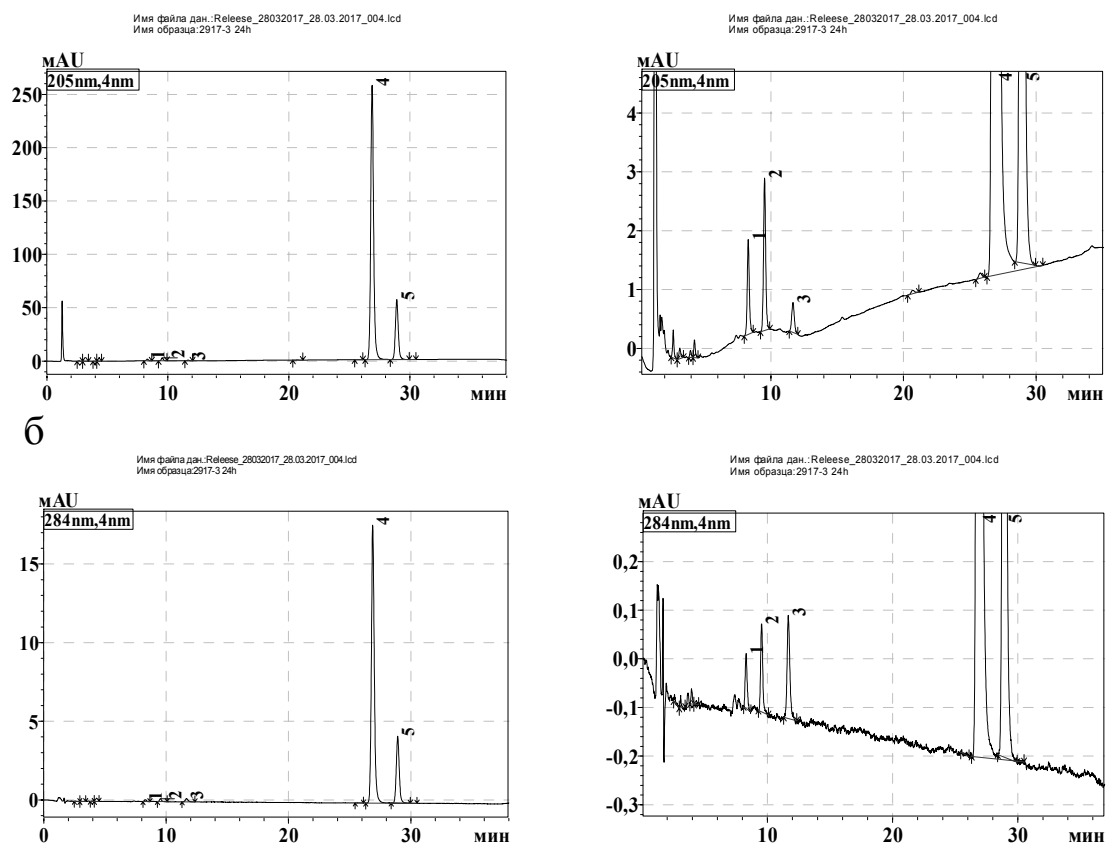

Рис. 2. Хроматограмма водного раствора этопозида (pH 7.4) при 205 нм (a) и 284 нм (б). 1-3, 5 - продукты разложения, 4-ЭТ. ПФ: линейный градиент $12 \rightarrow 25 \%$ $\mathrm{CH}_{3} \mathrm{CN}$ за 30 мин-0.5\% $\mathrm{HClO}_{4} \mathrm{pH} 7.0\left(\mathrm{NH}_{3}\right)$. Скорость потока ПФ $1.0 \mathrm{~cm}^{3} /$ мин. Колонка 4.6x20 мм+4.6x100 мм XBridge Shield RP18 3.5 мкм при $40^{\circ} \mathrm{C}$. Детектирование при 205 нм. Объем пробы 5 мкл. Температура образцов $5^{\circ} \mathrm{C}$.

Таблица 1. Характеристики чувствительности детектирования ЭТ и продуктов его разложения при различных длинах волн

\begin{tabular}{|c|c|c|c|}
\hline \multirow{2}{*}{ №№ пиков } & \multicolumn{2}{|c|}{$\begin{array}{c}\text { Соотношение сигнал/шум для пика этопо- } \\
\text { зида при длинах волн }\end{array}$} & \multirow{2}{*}{ Выигрыш в чувстви- } \\
\cline { 2 - 3 } & 205 нельности 205/284 нм & 284 нм & 21 \\
\hline 1 & 170 & 8 & 19 \\
\hline 2 & 270 & 14 & 3 \\
\hline 3 & 50 & 16 & 19 \\
\hline 4 (этопозид) & 26600 & 1400 & 19 \\
\hline 5 & 5800 & 300 & \\
\hline
\end{tabular}

Зависимость удерживания ЭТ от рН ПФ практически отсутствует, поскольку диссоциация фенольного гидроксила в его молекуле происходит только при $\mathrm{pH} 9.8$ [1]. Это не означает, что условия анализа ЭТ и его препаратов равноценны при любом значении $\mathrm{pH}$ буферной составляющей ниже указанного значения, поскольку в пробах могут содержаться продукты разложения ЭТ с отличными от исходного вещества физико-химическими и, соответственно, хроматографическими свойствами. Растворы ЭТ подвергали воздействию стресс-факторов, устанавливали фактор, наиболее критичный с точки зрения стабильности ЭТ, и для соответствующего раствора подбирали условия разделения ЭТ и продуктов его разложения.

Зависимость удерживания ЭТ от концентрации перхлората в ПФ не проявляется, что подтверждает неионизованное состояние молекулы в изученном диапазоне $\mathrm{pH}$. Однако, при исключении $\mathrm{ClO}_{4}^{-}$из ПФ наблюдается уширение пика ЭТ примерно 
в два раза, а его разделение с пиками продуктов разложения становится неполным (разрешение между соседними пиками $\mathrm{R}_{\mathrm{S}}<1$ ). Уже при концентрации $\mathrm{HClO}_{4}$ в растворе $0.1 \%$ эти факторы достигают оптимальных значений (ширина пика ЭТ на половине его высоты $\Delta t_{0,5} \approx 0.25$ мин, $\mathrm{R}_{S}>2$ ) и при дальнейшем увеличении концентрации буфера практически не изменяются.

Режим элюирования. При изменении концентрации $\mathrm{CH}_{3} \mathrm{CN}$ от 2 до 35 об. \% в течение 30 мин наблюдали нежелательный эффект - так называемый «эффект памяти» [22]. Этот эффект проявлялся появлением пика ЭТ на хроматограммах чистого растворителя. Нежелательный пик ЭТ наблюдали даже после промывки колонки $\mathrm{CH}_{3} \mathrm{CN}$ и начальным составом ПФ до стабилизации базовой линии; его площадь достигала 10 тыс мкВ×с при соотношении сигал/шум более 500 . Не приводила к положительному эффекту и промывка иглы и петли инжектора смесью (1:1) изопропанол-вода. «Эффект памяти» снижает точность количественного определения ЭТ и посторонних примесей при использовании в качестве стандарта разбавленного соответствующим образом раствора ЭТ. Поскольку изократическое элюирование не позволяет полностью разделить ЭТ и возможные продукты его разложения, была проведена оптимизация градиента. Уменьшение наклона градиента до $12 \rightarrow 25$ об. \% $\mathrm{CH}_{3} \mathrm{CN}$ за 30 мин не ухудшает разделение ЭТ и основных продуктов его разложения (табл. 2), но позволяет снизить проявление «эффекта памяти» до приемлемого минимума.

Таблица 2. Зависимость эффективности разделения ЭТ и продуктов его разложения от наклона используемого градиента

\begin{tabular}{|c|c|c|c|c|}
\hline \multirow{2}{*}{$\begin{array}{c}\text { Изменение концен- } \\
\text { трации ацетонитрила } \\
\text { за 30 мин. }\end{array}$} & $\begin{array}{c}\text { Прозуештыение пиков } \\
\text { разложения } \\
1 \text { и 2 }\end{array}$ & $\begin{array}{c}\text { Этопозид } \\
\text { и цис- } \\
\text { этопозид }\end{array}$ & $\begin{array}{c}\text { Коэффициент } \\
\text { асимметрии } \\
\text { пика этопозида }\end{array}$ & $\begin{array}{c}\text { Ширина пика это- } \\
\text { позида на половине } \\
\text { высоты, мин }\end{array}$ \\
\hline 1) От 2 до 35\% & 3.367 & 3.566 & 1.285 & 0.140 \\
\hline 2) От 7 до 30\% & 4.170 & 4.132 & 1.192 & 0.172 \\
\hline 3) От 12 до $25 \%$ & 4.080 & 4.824 & 1.156 & 0.245 \\
\hline
\end{tabular}

В связи с уменьшением высоты пиков при использовании более пологого градиента возможно снижение чувствительности. Однако, как было показано выше, этот показатель не уступает флуориметрическому и электрохимическому методам.

Стабильность ЭТ при различных условиях отражена выше в кратком обзоре литературы. Необходимость повторного исследования влияния основных стрессфакторов связана с принципиальным отличием состава и свойств используемой ПФ с буферной составляющей на основе $\mathrm{HClO}_{4}$, что обусловливает иные закономерности разделения ЭТ и продуктов его разложения.

Показано, что максимальная стабильность ЭТ в водных растворах обеспечивается в диапазоне рН 4-6, а при переходе в более кислые или более основные среды скорость разложения вещества значительно возрастает $[5,8,19]$. По силе воздействия на ЭТ стресс-факторы располагаются в следующем порядке: щелочь>кислота >окислитель>температура >УФ-излучение [14].

Окислитель. Проведенная работа в целом подтверждает литературные данные. Так, в смеси (1:9) ацетонитрил - 3\% водный раствор перекиси водорода при температуре $25^{\circ} \mathrm{C}$ ЭТ умеренно стабилен: в течение 3,5 часов его концентрация в растворе снижается на $3 \%$.

Кислота. В смеси (1:9) $\mathrm{CH}_{3} \mathrm{CN}-2 \%$ водный раствор $\mathrm{HClO}_{4}$ при $5^{\circ} \mathrm{C}$ площадь пика ЭТ за 2 ч. 40 мин снижается на 1\%. В дальнейшем при комнатной температуре 
в течение трех суток ЭТ в растворе разлагается практически полностью. Природа образующихся продуктов разложения в этом случае иная, чем при воздействии $\mathrm{H}_{2} \mathrm{O}_{2}$, поскольку времена удерживания этих продуктов другие. Следовательно, в изученных растворах $\mathrm{HClO}_{4}$ действует, прежде всего, как кислота.

Щелочь. Максимально быстрое разложение ЭТ наблюдается в щелочных растворах. При растворении в смеси (1:9) $\mathrm{CH}_{3} \mathrm{CN}-0.2 \mathrm{M} \mathrm{NaOH}$ практически сразу происходит полное разложение вещества, пик ЭТ ( $\mathrm{t}_{\text {уд }}$ около 26.5 мин) на хроматограмме отсутствует. В менее жестких условиях (смесь (1:9) $\mathrm{CH}_{3} \mathrm{CN}-1 \%$ водный раствор $\mathrm{NH}_{3}$ ) ЭТ разлагается медленнее, поэтому эти условия целесообразно использовать для изучения кинетики разложения и условий разделения ЭТ и продуктов его деструкции. Получаемые хроматограммы могут быть также использованы для подтверждения пригодности хроматографической системы для анализа ЭТ (рис. 3).

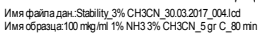

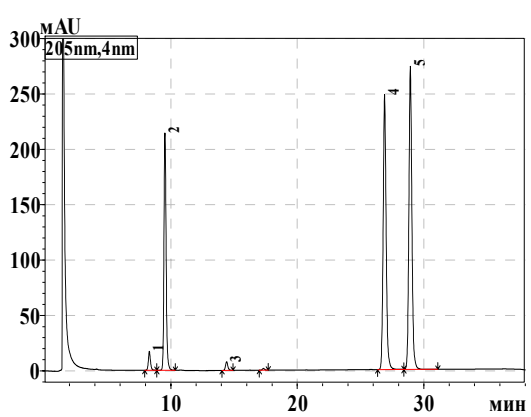

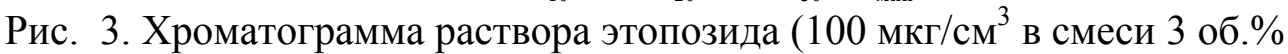
$\mathrm{CH}_{3} \mathrm{CN}-1 \%$ водный раствор $\mathrm{NH}_{3}$ ) через 80 мин после приготовления. Условия хроматографирования и нумерация пиков аналогичны рис. 2.

Влияние концентрации ацетонитрила и диметилсульфоксида в испытуемом растворе на скорость разложения. Выбор оптимального растворителя для пробоподготовки лекарственного препарата может быть сделан на основании их влияния на стабильность ЭТ в растворах, а также влияния на форму пиков и их разделение. Во всех приготовленных растворах содержание $\mathrm{NH}_{3}$ поддерживали постоянным, равным 1 масс. \%.

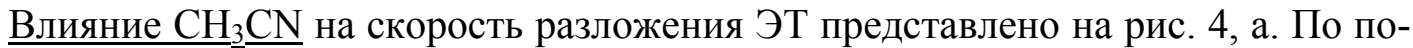
лученным данным видно, что добавление $\mathrm{CH}_{3} \mathrm{CN}$ в испытуемый раствор замедляет разложение ЭТ. Рассчитаны параметры линейной регрессии для зависимости натурального логарифма площади пика ЭТ от времени разложения с момента добавления $\mathrm{NH}_{3}$ в раствор. Коэффициент корреляции линейной зависимости $\mathrm{r}>0,99$. Коэффициент, характеризующий наклон линейной зависимости, численно равен значению константы скорости реакции разложения псевдопервого порядка. Рассчитанные значения константы скорости при разных значениях содержания $\mathrm{CH}_{3} \mathrm{CN}$ в растворах представлены в табл. 3.

Таблица 3. Зависимость константы скорости разложения ЭТ в $1 \%$ водном растворе $\mathrm{NH}_{3}$ от содержания $\mathrm{CH}_{3} \mathrm{CN}$ в растворе

\begin{tabular}{|c|c|}
\hline Концентрация $\mathrm{CH}_{3} \mathrm{CN}$, об. \% & Константа скорости разложения ЭТ, мин $^{-1}(\mathrm{P}=0.95 ; \mathrm{n}=4)$ \\
\hline 3.0 & $0.0112 \pm 0.0004$ \\
\hline 10.0 & $0.0078 \pm 0.0003$ \\
\hline 20.0 & $0.0039 \pm 0.0001$ \\
\hline 30.0 & $0.0023 \pm 0.0001$ \\
\hline
\end{tabular}

Голубицкий и др. / Сорбционные и хроматографические процессы. 2018. Т. 18. № 4 

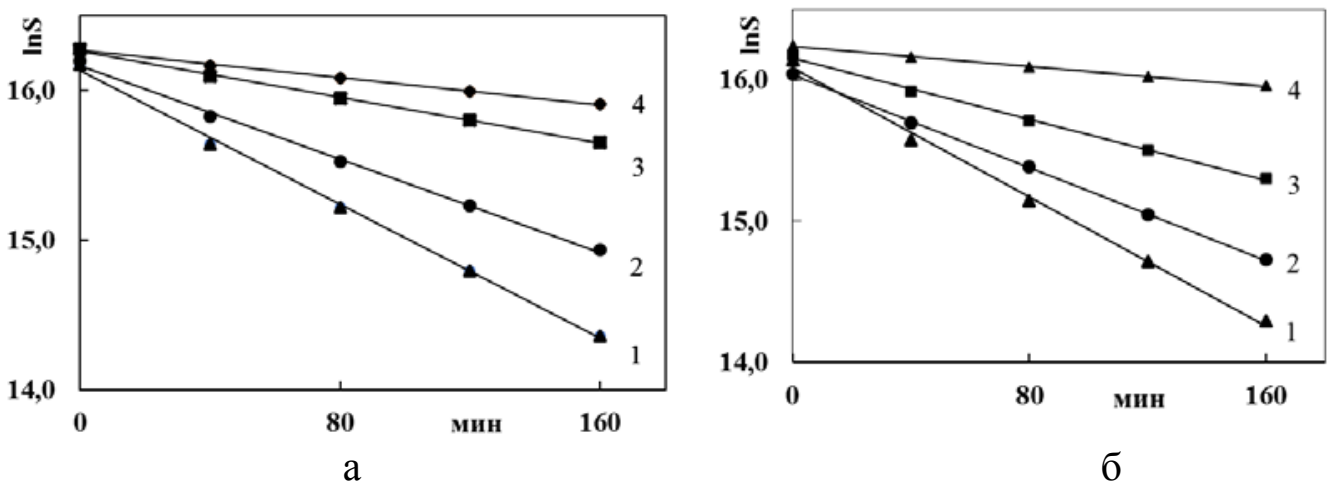

Рис. 4 Влияние концентрации, об. \% $\mathrm{CH}_{3} \mathrm{CN}$ (а) и об. \% ДМСО (б) на скорость разложения этопозида при $5^{\circ} \mathrm{C}$ в растворе $\left(100\right.$ мкг/ $\left.\mathrm{cm}^{3}\right)$, содержащем 1 масс. \% $\mathrm{NH}_{3}: 1-3 ; 2-10 ; 3-20 ; 4-30$ (а) и 50 (б).

В диапазоне содержаний 3-20 об.\% $\mathrm{CH}_{3} \mathrm{CN}$ ухудшение характеристик формы пиков и их разделения незначительно. При 30 об.\% значительно ухудшаются форма и разделение пиков основных продуктов разложения ЭТ, элюирующихся раньше основного вещества (табл. 4).

В соответствии с этими результатами оптимальным содержанием $\mathrm{CH}_{3} \mathrm{CN}$ в испытуемых растворах можно считать 20 об. \%, что обеспечивает стабильность ЭТ в растворе и характеристики хроматограмм, необходимые для получения точных количественных результатов.

Влияние ДМСО на скорость разложения ЭТ в растворах, содержащих 1 масс.\% $\mathrm{NH}_{3}$, представлено на рис. 4, б. По этим результатам видно, что ДМСО, как и $\mathrm{CH}_{3} \mathrm{CN}$, замедляет реакцию разложения ЭТ в щелочной среде, однако это влияние слабее. В отличие от $\mathrm{CH}_{3} \mathrm{CN}$, вплоть до содержания в растворе 50 об. \% влияние ДМСО на форму пиков ЭТ и продуктов его разложения и их разрешение пренебрежимо мало (табл 5).

Схема разложения этопозида в водно-органических растворах. Предложена схема разложения ЭТ в кислых и щелочных растворах, согласно которой и в кислой, и в щелочной среде процесс идет в две основные стадии. В кислой среде на первой стадии происходит отщепление гликозидного остатка молекулы (образование агликона ЭТ), на второй - образование транс-гидроксикислоты агликона ЭТ. В щелочной среде образование агликона ЭТ не происходит, но идет изомеризация - на первой стадии ЭТ переходит в цис-форму, на второй - образуется цис-гидроксикислота ЭТ [19], рис. 5.

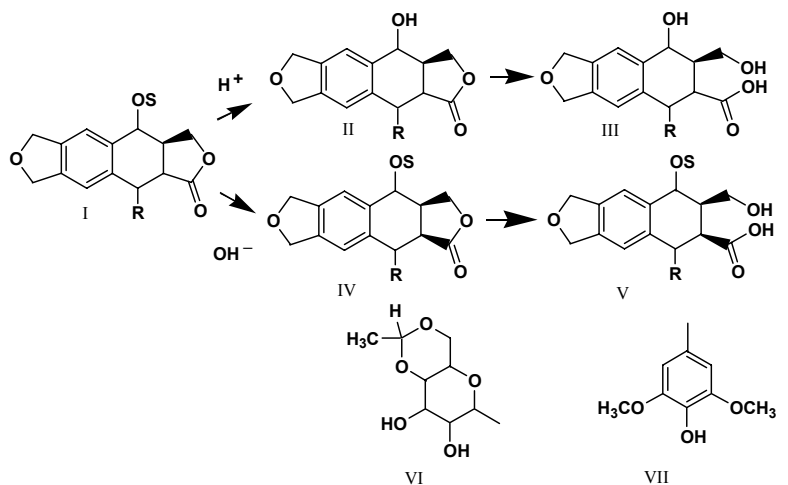

Рис. 5. Схема превращений этопозида в кислой или щелочной средах:

I - этопозид; II - агликон этопозида; III - транс-гидроксикислота агликона этопозида; IV - цис-этопозид; V - цис-гидроксикислота этопозида;

VI - гликозидный остаток (S); VII - радикал R. 
Результаты, полученные нами, подтверждают приведенную схему. На хроматограммах, полученных после разложения ЭТ в нейтральной и щелочной средах, присутствуют пики трех основных продуктов разложения, удерживаемых слабее ЭТ (пики 1-3 на рис. 2 и 3). Под воздействием кислоты ЭТ образует иные продукты разложения с другими временами удерживания при указанных условиях разделения. Пиков, удерживаемых сильнее основного вещества, в этом случае нет.

Согласно [19], цис-этопозид образуется только в щелочной среде. На хроматограмме раствора, содержащего 1 масс.\% аммиака (рис. 3), присутствует только один пик продукта со временем удерживания, превышающим время удерживания ЭТ (пик 5 на рис. 2 и 3). Можно предположить, что из всех продуктов превращения ЭТ в щелочной среде только цис-этопозид может иметь удерживание больше исходного вещества, поскольку при его образовании лишь частично изменяется конфигурация молекулы, но не изменяются ее размеры. Кроме того, по хроматограммам, полученным через разные промежутки времени после приготовления раствора видно, что этот пик растет в начале процесса, а впоследствии уменьшается, переходя в пики продуктов разложения, удерживаемые слабее. Согласно [19], в щелочной среде цисэтопозид является промежуточным продуктом разложения ЭТ, переходя со временем в цис-гидроксикислоту ЭТ.

Кислотная природа продуктов разложения ЭТ подтверждается сравнительным элюированием раствора ЭТ с использованием ПФ с нейтральной (pH 7.0) и кислой (рН 2.5) буферными составляющими. В растворах, получаемых при извлечении ЭТ из полимерных наночастиц фосфатным буферным раствором с $\mathrm{pH} 7.4$, также присутствуют пики цис-этопозида (5) и основных продуктов разложения (1-3) (рис. 2), по временам удерживания и спектрам поглощения соответствующие пикам, наблюдаемым на хроматограммах растворов ЭТ, содержащих 1 масс.\% аммиака (рис. 3). При переходе к кислотной ПФ время удерживания основного продукта разложения ЭТ (2) возрастает примерно в два раза, что подтверждает его переход из диссоциированной в молекулярную форму, что характерно для кислоты.

Зависимость скорости разложения ЭТ от содержания органических растворителей объясняется следующими закономерностями. Известно, что скорость реакции между ионами одного знака растет при увеличении диэлектрической постоянной раствора [23]. Значение рК ЭТ равно 9.8 [1], следовательно, в растворах, содержащих 1 масс.\% аммиака (pH 11.5) его молекула диссоцирована и имеет отрицательный заряд. Отрицательно заряжен и второй участник реакции - гидроксил-ион. Следовательно, при снижении содержания органического растворителя в водном растворе, т.е. росте диэлектрической постоянной, скорость реакции должна расти. Результаты проведенных исследований подтвердили данное предположение. Диэлектрическая проницаемость $\mathrm{CH}_{3} \mathrm{CN}$ ниже, чем у ДМСО [24], следовательно, его добавление должно в большей степени замедлять реакцию разложения ЭТ. Это также подтверждается полученными результатами. ДМСО - более полярный растворитель по сравнению с $\mathrm{CH}_{3} \mathrm{CN}$, поэтому его элюирующая способность в обращенно-фазной ВЭЖХ ниже. По этой причине его добавление в испытуемые растворы должно меньше влиять на размывание пиков, что также подтверждается экспериментально.

\section{Заключение}

ЭТ нестоек в щелочной среде, что это необходимо учитывать при разработке состава, технологии лекарственных препаратов и методик анализа. Добавление в растворы ЭТ ацетонитрила или ДМСО замедляет разложение этого вещества в щелочной среде. ДМСО более предпочтителен для пробоподготовки препаратов ЭТ,

Голубицкий и др. / Сорбционные и хроматографические процессы. 2018. Т. 18. № 4 
поскольку его растворяющая способность выше, а искажающее воздействие на форму пиков ниже, чем у ацетонитрила. Полученные результаты могут быть использованы при анализе пролонгированной лекарственной формы ЭТ и разработке методик анализа других лекарственных средств.

Растворы ЭТ, содержащие продукты разложения, целесообразно анализировать в градиентном режиме при изменении содержания ацетонитрила в ПФ от 12 до 25 об. \% в течение 30 мин, используя в качестве буферного компонента ПФ водный раствор хлорной кислоты (0.3-0.5 об. \%) с добавлением аммиака до $\mathrm{pH} 6.7-7.3$. Такой подход позволяет полностью разделить анализируемые вещества и повысить чувствительность определения за счет детектирования в коротковолновой области УФ-спектра.

\section{Список литературы}

1. Etoposide. Open Chemistry Database (PubChem). Compound Summary for CID 36462. U.S. National Library of Medicine National Center for Biotechnology Information.

2. Delmas A., Gordien J. B., Bernadou J. M., Roudaut M., Gresser A. // J. Pharm. Biomed. Anal. 2009. Vol. 49. No 5. pp. 1213-1220.

3. Zhang T., Shen Q., Pan W. // Chromatographia. 2010. Vol. 71. N. 11/12. P. 993-998.

4. The United States Pharmacopoeia. The National Formulary (USP36 - NF31). 2013. Vol. 2. pp. 3523-3526.

5. Tian L., He H., Tang X. // J. Pharm. Sci. 2007. Vol. 96. No 7. pp. 1719-1728.

6. Eisenberg E.J., Eickhoff W.M. // J. Chromatogr. B. 1993. Vol. 621. No 1. pp. 110-114.

7. Chevrier R., Sautou V., Pinon V., Demeocq F., Chopineau J. // Pharm. Acta Helvet. 1995. Vol. 70. No 2. pp. 141-148.

8. Chow D. Shan J. // J. Chromatogr. 1987. Vol. 396. pp. 217-223.

9. Liliemark E., Pettersson B., Peterson C., Liliemark J. // J. Chromatogr. B. 1995. Vol. 669. No 2. pp. 311-317.

10. European Pharmacopoeia - 8-th edition (EP 8.0). 2013. Vol. 2. pp. 2202-2205.

11. Yin J., Yang Y., Li K., Zhang J., Shao B. // J. Chromatogr. Sci. 2010. Vol. 48. No 10. pp. 781-789.

12. Ferrando-Climent L., Rodriguez-Mozaz S., Barceló D.D. // Anal. Bioanal. Chem. 2013. Vol. 405. No 18. pp. 5937-5952.

13. Marti'n J., Camacho-Mun oz D., Santos J. L., Aparicio I., Alonso E. // J. Sep. Sci. 2011. Vol. 34. No 22. pp. 3166-3177.

14. Algan, A. H., Gumustas M., Karatas A., Ozkan S.A. // J. Pharm. Biomed. Anal. 2016. Vol. 124. pp. 382-389.

15. Stremetzne S., Jaehde U., Schunack W. // J. Chromatogr. B. 1997. Vol. 703. No 1-2. pp. 209-215.

16. Manouilov K. K., McGuire T. R., Gordon B. G., Gwilt P. R. // J. Chromatogr. B. 1998. Vol. 707. No 1-2. pp. 342-346.

17. Cai X., Woo M.H., Edick M.J., Relling M.V. // J. Chromatogr. B. 1999. Vol. 728. No 2. pp. 241-250.

18. Saadati R., Dadashzadeh S. // J. Liq. Chrom. Rel. Tech. 2011. Vol. 34. No 18. pp. 2130-2148.

19. Beijnen J.H., Holthuis J.J.M., Kerkdijk H.G., van der Houwen O.A.G.J. et al. // Int. J. Pharm. 1988. Vol. 41 No 1-2. pp. 169-178.

20. Jasti B.R., DuJ., Vasavada R.C. // Int. J. Pharm. 1995. Vol. 118. No 2. pp. 161-167.

21. Голубицкий Г.Б., Владимирова Е.В., Островский К.П., Гельперина С.Э. // Сорбиионные и хроматографические проиессы. 2017. T. 17. № 5. C. 772-780.

22. Kromidas S. Practical problem solving in HPLC / Weinheim: WILEY-VCH. 2004. 178 p. pp. 91-92.

23. Марк Г., Рехниц Г. Кинетика в аналитической химии. М. Мир. 1972. 368 с.

24. Рудаков О.Б., Востров И.А., Федоров С.В., Филлипов А.А., Селеменев В.Ф., Приданцев А.А. Спутник хроматографиста. Методы жидкостной хроматографии. Воронеж. Водолей. 2004. 528 с. 


\section{References}

1. Etoposide. Open Chemistry Database (PubChem). Compound Summary for CID 36462. U.S. National Library of Medicine National Center for Biotechnology Information.

2. Delmas A., Gordien J. B., Bernadou J. M., Roudaut M., Gresser A., J. Pharm. Biomed. Anal., 2009, Vol. 49, No 5, pp. 1213-1220.

3. Zhang T., Shen Q., Pan W., Chromatographia, 2010, Vol. 71, No 11/12, pp. 993-998.

4. The United States Pharmacopoeia. The National Formulary (USP36 - NF31), 2013, Vol. 2, pp. 3523-3526.

5. Tian L., He H., Tang X., J. Pharm. Sci., 2007, Vol. 96, No7, pp. 1719-1728.

6. Eisenberg E. J., Eickhoff W. M., J. Chromatogr. B., 1993, Vol. 621, No 1, pp. 110-114.

7. Chevrier R., Sautou Vol., Pinon Vol., Demeocq F., Chopineau J., Pharm. Acta Helvet, 1995, Vol. 70, No 2, pp. 141-148.

8. Chow D. Shan J., J. Chromatogr., 1987, Vol. 396, pp. 217-223.

9. Liliemark E., Pettersson B., Peterson C., Liliemark J., J. Chromatogr. B., 1995, Vol. 669, No 2, pp. 311-317.

10. European Pharmacopoeia - 8-th edition (EP 8.0), 2013, Vol. 2, pp. 2202-2205.

11. Yin J., Yang Y., Li K., Zhang J., Shao B., J. Chromatogr. Sci., 2010, Vol. 48, No 10, pp. 781-789.

12. Ferrando-Climent L., Rodriguez-Mozaz S., Barceló D., Anal. Bioanal. Chem., 2013, Vol. 405, No 18, pp. 5937-5952.

13. Mart1'n J., Camacho-Muñoz D., Santos J. L., Aparicio I., Alonso E., J. Sep. Sci., 2011, Vol. 34, No 22, pp. 3166-3177.

Голубицкий Григорий Борисович - старший научный сотрудник лаборатории систем доставки лекарственных средств, д.Х.н., ООО «Технология лекарств», Химки.

Островский Константин Петрович - научный сотрудник лаборатории систем доставки лекарственных средств, ООО «Технология лекарств», Химки.

Гельперина Светлана Эммануиловна - начальник лаборатории системдоставки лекарственных средств, д.х.н., ООО «Технология лекарств», Химки.
14. Algan, A.H., Gumustas M., Karatas A., Ozkan S.A., J. Pharm. Biomed. Anal., 2016, Vol. 124, pp. 382-389.

15. Stremetzne S., Jaehde U., Schunack W., $J$. Chromatogr. B, 1997, Vol. 703, No 1-2, pp. 209-215.

16. Manouilov K. K., McGuire T. R., Gordon B. G., Gwilt P.R., J. Chromatogr. B., 1998, Vol. 707, No 1-2, pp. 342-346.

17. Cai X., Woo M. H., Edick M. J., Relling M. V., J. Chromatogr. B., 1999, Vol. 728, No 2, pp. 241-250.

18. Saadati R., Dadashzadeh S., J. Liq. Chrom. Rel. Tech., 2011, Vol. 34, No 18. pp. 2130-2148.

19. Beijnen J. H., Holthuis J. J. M., Kerkdijk H. G., van der Houwen O. A. G. J. et al., Int. J. Pharm., 1988, Vol. 41,No 1-2, pp. 169-178.

20. Jasti B.R., Du J., Vasavada R.C., Int. J. Pharm., 1995, Vol. 118, No 2, pp. 161-167.

21. Golubitsky, G. B., Vladimirova E. Vol., Ostrovskiy K. pp., Gelperina S. E., Sorbtsionnye I khromatografichkie protsessy, 2017, Vol. 17, No 5, pp. 772-780.

22. Kromidas S. Practical problem solving in HPLC / Weinheim: WILEY-VCH. 2004. 178 p. pp. 91-92.

23. Mark G., Rechnitz G. Kinetics in analytical chemistry,M., Mir, 1972, 368p. pp. 20-21.

24. Rudakov O.B., Vostrov I.A., Fedorov S.Vol., Filippov A.A., Selemenev Vol.F., Pridantsev A. A. The satellite of chromatographer. Methods of liquid chromatography, Voronezh, AVodoley, 200,. 528 p. pp. 295-296.

Golubitsky, Grigory B. - Senior researcher of the laboratory delivery systems of medicines, grand Ph.D Chemistry, LTD "Drugs Technology", Khimki, e-mail: iirogirg@narod.ru

Ostrovsky Konstantin P. - Researcher of the laboratory of delivery systems of medicines, LTD "Drugs Technology", Khimki.

Gelperina Svetlana E. - Head of the laboratory of delivery systems of medicines, grand Ph.D Chemistry, LTD “Drugs Technology”, Khimki. 